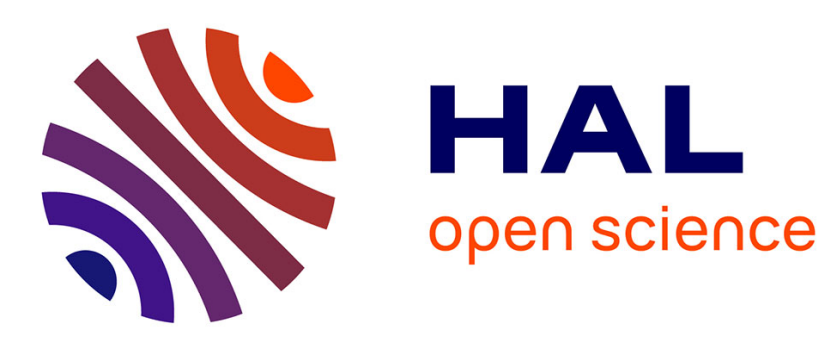

\title{
Analysis of pressure fluctuations in swirling turbulent flows
}

\author{
Patrice Abry, S. Fauve, P. Flandrin, C. Laroche
}

\section{To cite this version:}

Patrice Abry, S. Fauve, P. Flandrin, C. Laroche. Analysis of pressure fluctuations in swirling turbulent flows. Journal de Physique II, 1994, 4 (5), pp.725-733. 10.1051/jp2:1994101 . jpa-00247995

\section{HAL Id: jpa-00247995 https://hal.science/jpa-00247995}

Submitted on 1 Jan 1994

HAL is a multi-disciplinary open access archive for the deposit and dissemination of scientific research documents, whether they are published or not. The documents may come from teaching and research institutions in France or abroad, or from public or private research centers.
L'archive ouverte pluridisciplinaire HAL, est destinée au dépôt et à la diffusion de documents scientifiques de niveau recherche, publiés ou non, émanant des établissements d'enseignement et de recherche français ou étrangers, des laboratoires publics ou privés. 
Classification

Physics Abstracts

$47.25 \mathrm{M}-47.30$

\title{
Analysis of pressure fluctuations in swirling turbulent flows
}

\author{
P. Abry, S. Fauve, P. Flandrin and C. Laroche
}

Laboratoire de Physique, CNRS-URA 1325, Ecole Normale Supérieure de Lyon, 46 Allée d'Italie, 69364 Lyon, France

(Received 10 December 1993, accepted 21 January 1994)

\begin{abstract}
Turbulent flows display intermittent pressure drops associated with vorticity concentrations. Using wavelet transform, we characterize these low pressure events and study some of their statistical characteristics in a flow generated between the gap of two counterrotating disks. We show that the mean frequency of intermittent pressure drops decreases with increasing Reynolds number.
\end{abstract}

\section{Introduction.}

Pressure fluctuations in turbulent flows have been studied for a long time [1-4], but it has been emphasized only recently [5] that pressure can be used to locate regions with high vorticity or dissipation in turbulent flows. We have shown by direct measurement of the pressure in the flow between two counter-rotating disks, that pressure fluctuations display random occurrence of strong pressure drops which correspond to vorticity concentrations [6]. This generates an asymmetric probability density function (PDF), with a roughly exponential tail for low pressures whereas the high pressure cut-off is sharper. We study here some statistical characteristics of the pressure drops. We first determine the mean duration of these events. We then study the shape of the pressure PDF as a function of the Reynolds number and we check the effect of the size of the pressure transducers and of the frequency-filtering of the pressure signal. Finally, we consider the contribution of the strongest pressure drops to the pressure PDF and power spectrum. To wit, we remove them from the pressure signal using an orthogonal wavelet decomposition, and show that their contribution to the power spectrum is mostly at frequencies smaller than the integral scale, i.e. the inverse rotation rate of the disks. Finally we consider statistics of waiting times between two successive pressure drops, and show that the mean frequency of these intermittent events decreases with increasing Reynolds number. 


\section{Experimental measurements and signal processing technique.}

The experimental set-up of reference [6] has been slightly modified in order to reach higher Reynolds numbers. It consists of a cylindrical container, $19.3 \mathrm{~cm}$ in inner diameter and $19.8 \mathrm{~cm}$ in height, filled with water or with a water-glycerol mixture in order to change the Reynolds number at a fixed rotation rate. Two co-axial rotating disks of diameter $D=19 \mathrm{~cm}$, at a distance $H=8 \mathrm{~cm}$ apart from each other, are rotated independently at angular velocities $\Omega_{1,2}$ in the range $[0,4000] \mathrm{rpm}$, i.e. $[0,67] \mathrm{Hz}$ for $\Omega_{1,2} / 2 \pi$. In a series of experiments radial blades have been fixed on the disks. This increases the efficiency of the stirring ( $V_{\mathrm{rms}}$ is increased roughly by a factor 5) but the results presented here for the pressure are qualitatively unchanged.

Pressure fluctuations are measured by piezoelectric transducers, mounted flush with the lateral wall. Their diameter $\Phi$ are respectively, 15.7, 11, 5.5 and $2.6 \mathrm{~mm}$. They are acceleration-
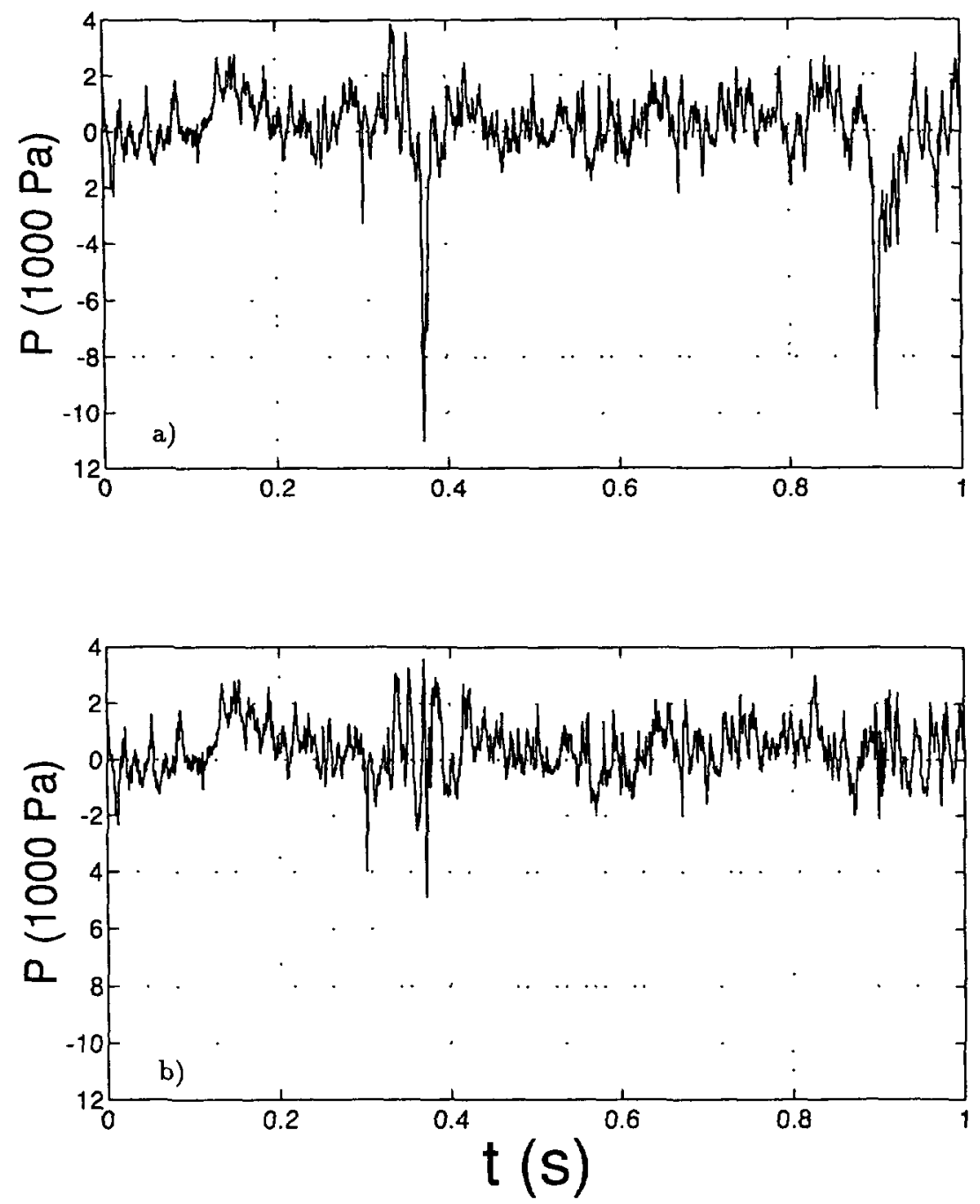

Fig. 1. - a) Direct time-recording of the pressure. $\Omega=2000 \mathrm{rpm}, \nu=0.006 \mathrm{~cm}^{2} / \mathrm{s}, \Phi=5.5 \mathrm{~mm}$. b) Same as (a), but the pressure drops have been cancelled using a DWT technique. 
compensated except the smallest one. Their low frequency cut-off at $-3 \mathrm{~dB}$ is $50 \mathrm{mHz}$ for the two large ones and $15 \mathrm{mHz}$ for the small ones; their rise-time are respectively, 8, 5, 1, $1 \mu \mathrm{s}$. Although their electric sensitivities are different, they give the same amplitude of pressure within $2 \%$ when calibrated.

A direct time-recording of pressure displays asymmetric fluctuations with random occurrence of strong pressure drops (Fig. 1a). In order to characterize and detect these events in a systematic way [7], we have used a discrete wavelet transform (DWT) [8]. The key point of this wavelet-based detection is that the pressure drops (which are pulse-like shaped) involve a large range of scales (or frequencies) and, thus generate a DWT with large detail-coefficients on many different scales. The event detection technique then consists in tracking time coincidences in detections performed independently on different scales [7]. Spurious fluctuations, on the contrary, are uncorrelated and thus do not coincide in time at different scales. A concurrent detection method based on a direct threshold overstepping yields very similar lists of event locations. We then compare the statistical properties of the original pressure recording to that of a very similar signal where the pressure drops have been cancelled. Using an orthonormal discrete wavelet expansion of the data (with a Daubechies-3 wavelet) allows to replace at each scale, the detail-coefficient coinciding in time with a pressure drop, by what it would have been if the signal had behaved as it does before and after the pressure drop (i.e. by an interpolation of the previous and following detail-coefficients). Here, the use of an orthonormal expansion is of major importance since it allows the modification of a single detail-coefficient independently from its neighbours. The wavelet reconstruction of the signal is displayed in figure $1 \mathrm{~b}$; one can check that the strong pressure drops have been eliminated.

Moreover, the signal is not modified except in the immediate vicinity of these events and no extra frequencies have been introduced; this would have been impossible with any frequency filtering or with a sharp cut-off of the low pressures in the recording.

\section{Duration of the pressure drops.}

The width in time $\tau$ of each pressure drop is measured at half of the maximum pressure drop. The histograms, computed on about 6000 successive events for different rotation rates and transducer sizes, display a sharp peak which gives the most probable value $\tau_{\mathrm{m}}$ (see Tab. I).

Table I. - Most probable value $\tau_{\mathrm{m}}$ of the duration of the pressure drops for different rotation rates $\Omega$ and different sizes of the transducers $(\Phi=15.7 \mathrm{~mm}(1)$ and $\Phi=5.5 \mathrm{~mm}(2)) . V=\Phi / \tau_{\mathrm{m}}$. $P_{\mathrm{rms}}$ is the rms value of pressure fluctuations.

$\begin{array}{llll}\Omega(\mathrm{rpm}) & 400 & 500 & 800 \\ \tau_{\mathrm{m}}(\mathrm{ms})(1) & 12.5 & - & - \\ \tau_{\mathrm{m}}(\mathrm{ms})(2) & 4.8 & 3.9 & 2.5 \\ V(\mathrm{~m} / \mathrm{s}) & 1.5 & 1.8 & 5.5 \\ \sqrt{P_{\mathrm{rms}}} / V & 0.2 & 0.17 & 0.2\end{array}$

We observe that the charateristic time-scale of pressure drops is roughly proportional to the transducer size. This shows that these events consist of a spatially localized pressure drop sweeping on the transducers with a mean velocity $V$, which increases with the rotation rate. 
$V$ is estimated as the ratio of the transducer size to $\tau_{m}$; we also measure the rms value of pressure fluctuations and find that $\sqrt{P_{\mathrm{rms}}} / V$ is roughly constant as can be infered by the relation $P_{\mathrm{rms}} \propto \rho V_{\mathrm{rms}}^{2}[3]$.

\section{Shape of the pressure PDF.}

The PDFs of pressure fluctuations recorded for $\Omega=2000 \mathrm{rpm}$ with transducers of different sizes in water and in a water-glycerol mixture are displayed in figure 2 . As already noticed, pressure fluctuations are strongly asymmetric. The pressure drops generate a roughly exponential tail toward low pressures. However, it is visible that the tail is stretched with the smaller transducer
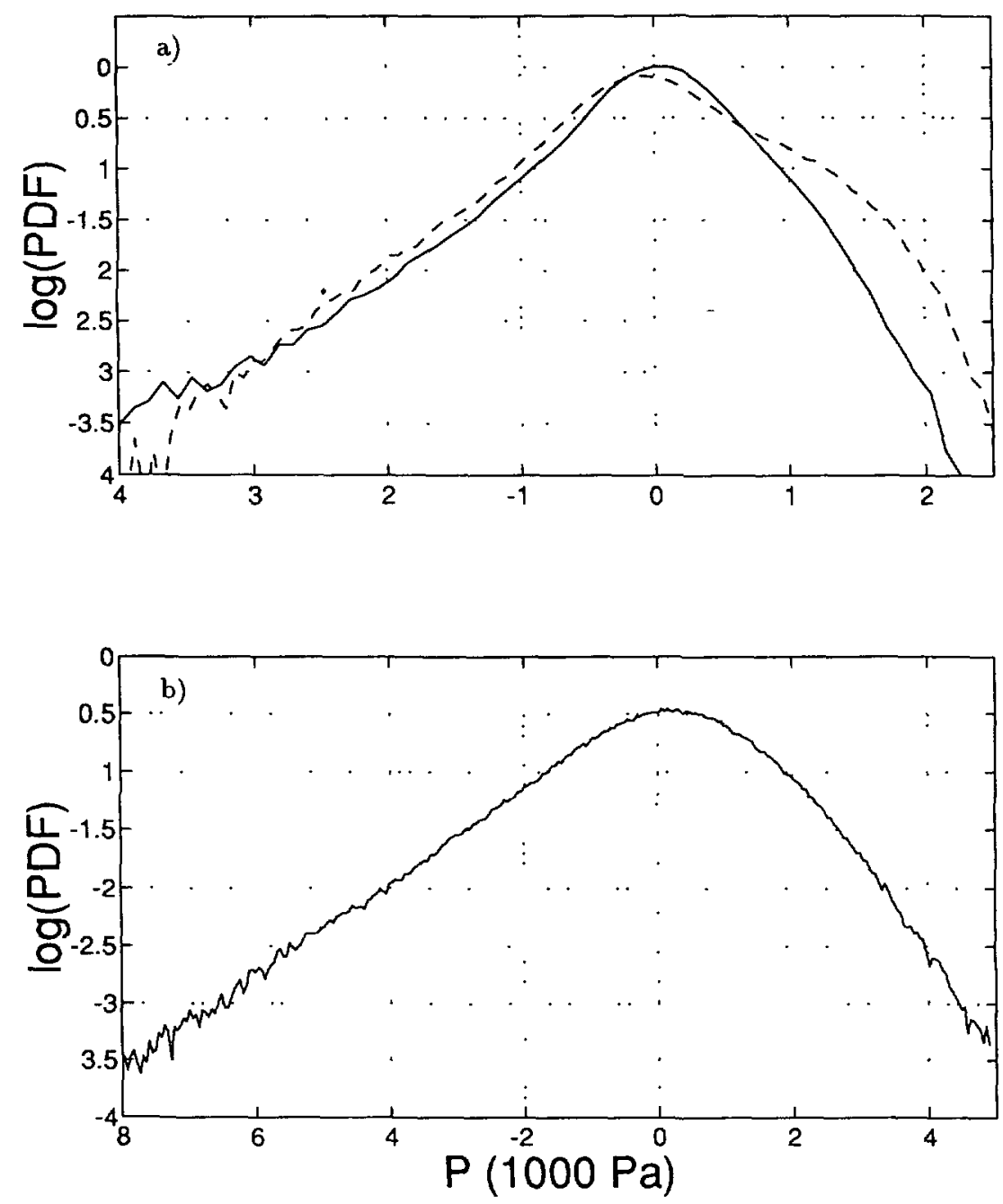

Fig. 2. - a) Pressure PDFs for $\Omega=2000 \mathrm{rpm}$ and $\nu=0.005 \mathrm{~cm}^{2} / \mathrm{s}$ (water); the diameter of the transducer is $\Phi=15.7 \mathrm{~mm}$ (dashed line), $\Phi=5.5 \mathrm{~mm}$ (full line). b) Pressure PDF for $\Omega=2000 \mathrm{rpm}$ and $\nu=0.2 \mathrm{~cm}^{2} / \mathrm{s}$ (glycerol-water mixture), $\Phi=5.5 \mathrm{~mm}$. 
( $5.5 \mathrm{~mm}$ ), i.e. displays an upward concavity in the log-lin plot of figure 2a (full line). This is also observed with the smallest transducer $(2.6 \mathrm{~mm}$ in diameter) whereas the 11 and $15.7 \mathrm{~mm}$ ones display a nearly exponential tail. We also notice that even with the small transducers, the tail is exponential at smaller Reynolds number (Fig. 2b); this is in agreement with the behavior observed in numerical simulations [9].
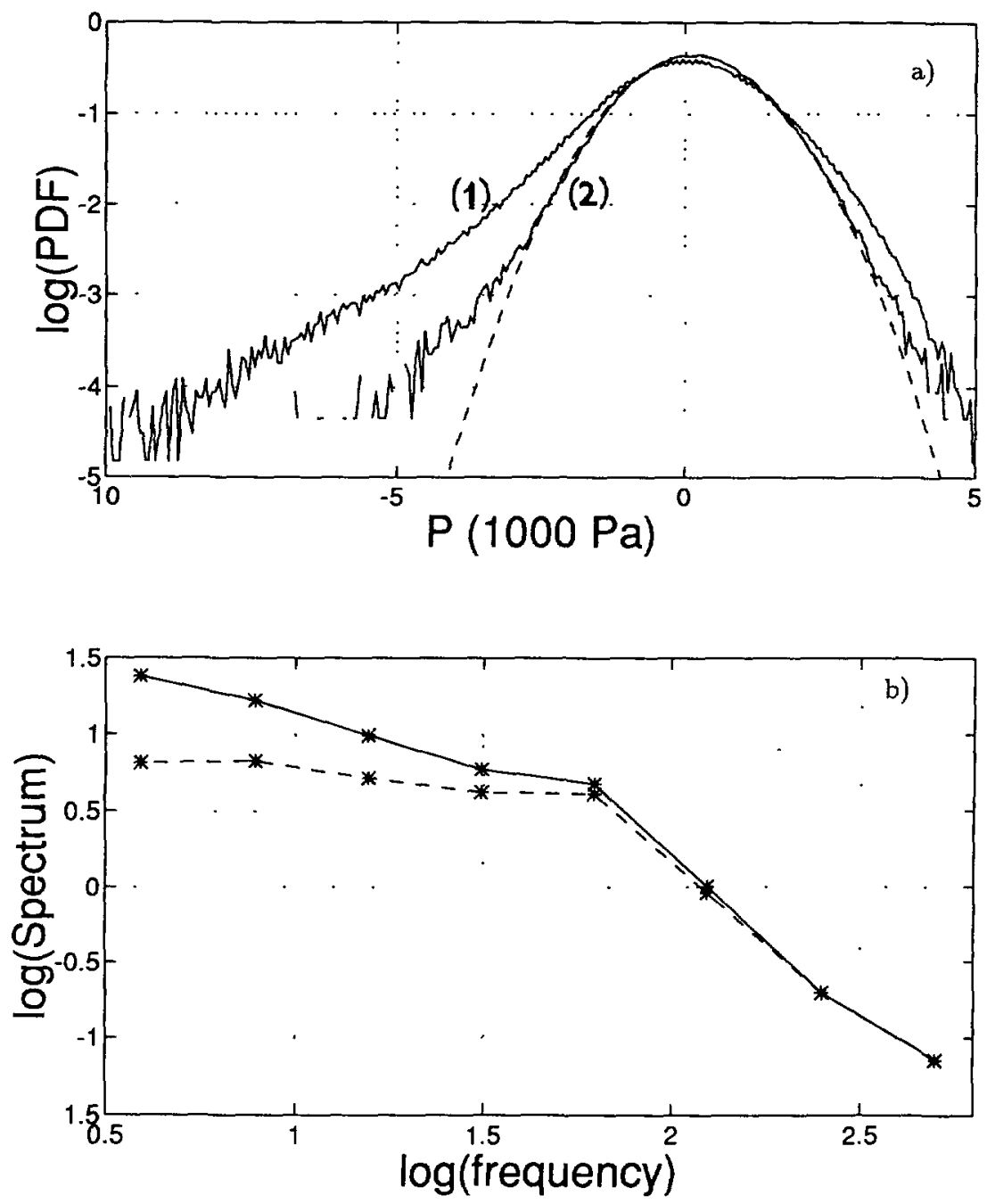

Fig. 3. - a) (1) Pressure PDF for $\Omega=800 \mathrm{rpm}$ and $\nu=0.006 \mathrm{~cm}^{2} / \mathrm{s}$ (water; disks with radial blades); (2) same as (1) but the pressure drops have been removed using a DWT technique; (dashed line) Gaussian fit of (2). b) Time-scale based estimation of the corresponding power spectra: (full line) direct pressure signal (1); (dashed line) pressure signal after cancellation of the pressure drops (2).

By frequency-filtering the pressure signal, we have checked that the shape of the PDFs is not affected by the low and high-frequency cut-off of the transducers. On the contrary, as shown above, there is a clear size effect of the transducers. This does not result directly from 
their spatial resolution. Indeed, it is known $[9,10]$ that the characteristic size of vorticity filaments is about 5 times the Kolmogorov length $\eta$, i.e. about $100 \mu \mathrm{m}$ in our experiments, which is very small compared to all the transducers. However, it is also known that the typical life-time of the filaments is the integral time-scale $[9,10,12]$. Consequently, they travel a typical distance $2 \pi V / \Omega$, i.e. several $\mathrm{cm}$ in our experiments, during their life-time. Thus, one can explain the effect of the transducer size as follows: the filaments are not detected as frozen structures sweeping on the transducers, and their own time-evolution affects pressure measurements, especially with large transducers.

To characterize further the low pressure events, we have cancelled them from the signal using the wavelet technique described above, and we have compared the PDFs and the pressure spectra of the original and resynthetized signals (Fig. 3). The PDF of the resynthetized signal is roughly Gaussian, but still slightly skewed to low pressures. Let us emphasize that the wavelet technique does not involve any a priori information (amplitude or sign of the pressure fluctuations) as would be the case for a threshold-based detection. Therefore the above result is not an obvious consequence of the detection method. The time-scale-based estimation of the power spectra [11] are displayed in figure 3b. Although the pressure drops involve all the frequencies present in the whole signal, their contribution is larger at low frequency, i.e. at the integral time-scale.

\section{Waiting times.}

The PDF of the waiting time $t$ between two successive pressure drops, is displayed in figure 4. One observes a power law for small times followed by an exponential decay at larger times. The cross-over occurs for a waiting time $t_{\mathrm{c}}$ which varies in the range $[0.1,1] \mathrm{s}$ as the Reynolds number is varied. The shape of the PDF is unchanged but $t_{c}$ decreases with increasing Reynolds number. Note that $t_{\mathrm{c}}$ is larger than the integral time-scale $2 \pi / \Omega$, thus the corresponding length $V t_{c}$ is larger than the integral scale. Consequently, the departure from a Poisson law at small waiting times does not result from the finite size of the transducers; similarly, it is unlikely that it results from the detection of several pressure minima due to a twisted or bursting filament, sweeping on the transducer. The value of $t_{\mathrm{c}}$ indicates that successive pressure drops can be considered as independent events only when the waiting time is large compared to the integral time-scale.

The dimensionless mean waiting time, $\Omega \bar{t} / 2 \pi$, is displayed as a function of the Reynolds number in figure 5 , showing that the mean frequency of pressure drops decreases when the Reynolds number is increased.

\section{Discussion.}

The low pressure tail of the pressure PDF results from pressure drops due to vorticity concentrations [6]. However, it has been shown numerically [13] and analytically [14] that a Gaussian velocity field gives a skewed pressure-PDF with exponential tails. This may appear puzzling since vorticity filaments are not observed in a Gaussian velocity field. In fact, this approximation is not a good one to evaluate pressure fluctuations which are strongly underestimated 

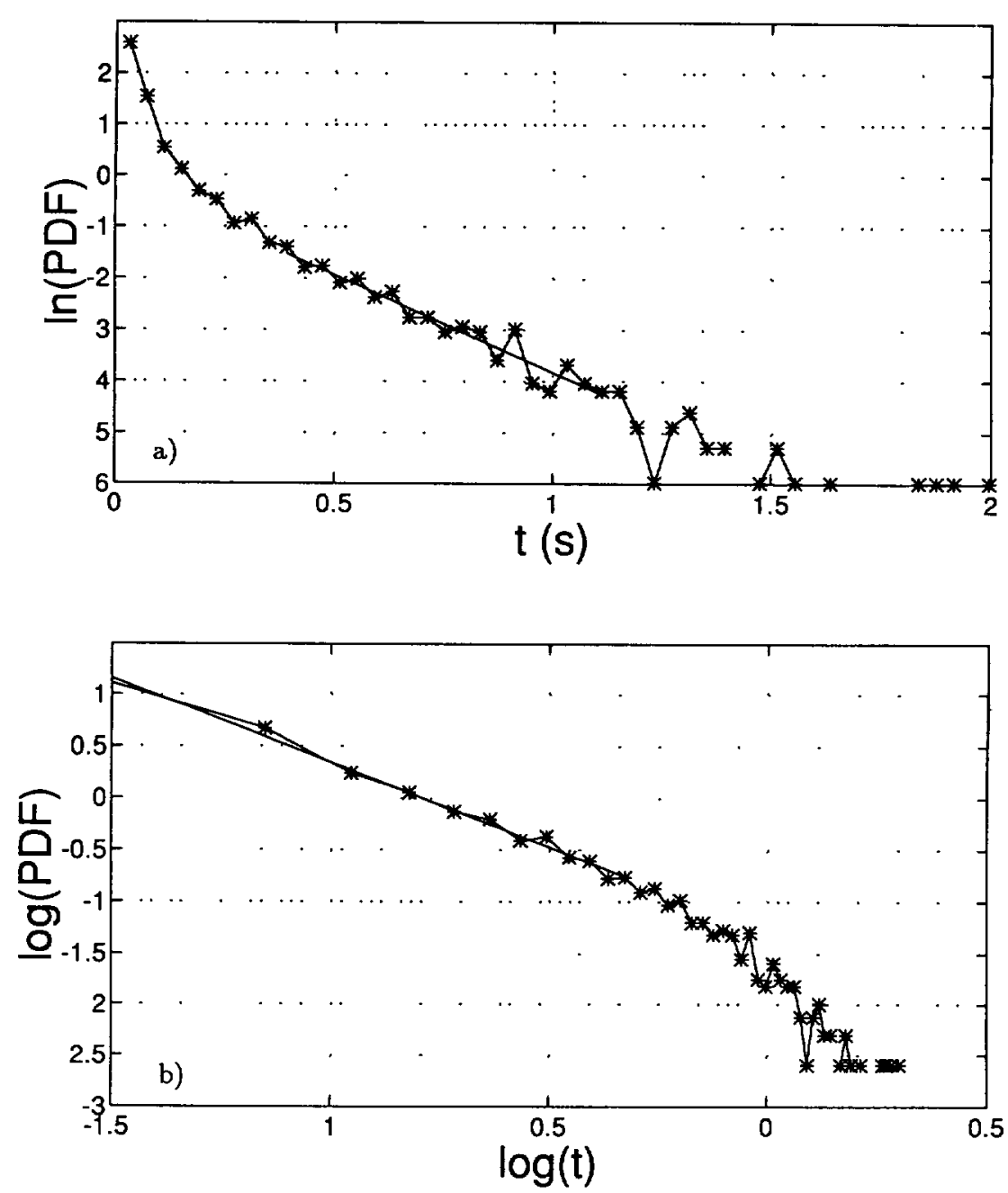

Fig. 4. - PDF of the waiting time between two successive pressure drops; $\Omega=800 \mathrm{rpm}$ and $\nu=$ $0.006 \mathrm{~cm}^{2} / \mathrm{s}$ (water; disks with radial blades): (a) $\log$-lin plot, (b) $\log -\log$ plot.

[9]. The high pressure part of the PDF was fitted to $\left(P_{\max }-P\right)^{4}$ in reference [6]. However, a Gaussian fit is equally good and even better with the smallest pressure probes; this behavior is not understood theoretically, even with a simple model.

The PDFs of $P / \Omega^{2}$ for different $\Omega$ s roughly collapse on the same curve [6], showing that pressure fluctuations are mostly generated by velocity at the integral scale. This is not in disagreement with the fact that vorticity filaments do generate the strongest pressure fluctuations, except if one considers them as small scale structures; this is however a wrong concept since vorticity filaments involve kinetic energy at all scales. We have shown that their contribution to the pressure also involves all the scales of the flow, and is stronger at large scales [15]. Pressure measurements show that they involve a characteristic velocity difference which is roughly the integral velocity, and the statistics of waiting times show that they display some correlation on the integral time-scale. All these characteristics are the ones of large scales. It 


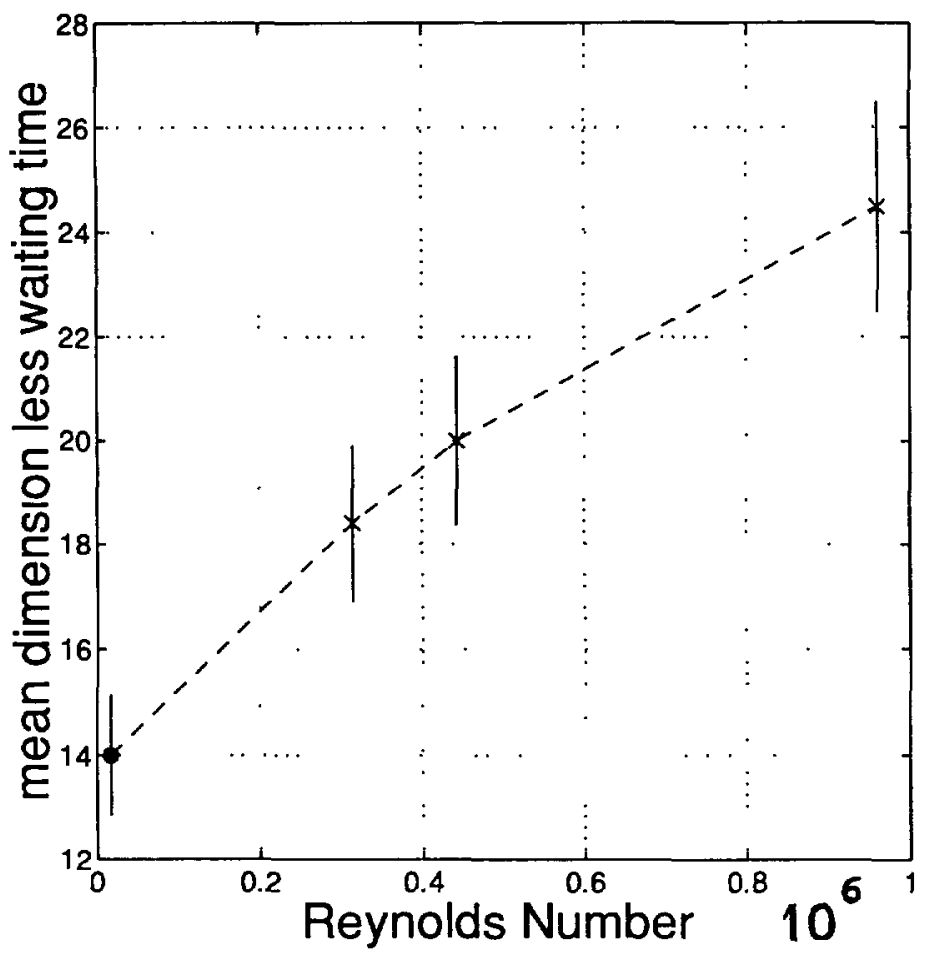

Fig. 5. - Mean dimensionless waiting time $\Omega \bar{t} / 2 \pi$ between two successive pressure drops, as a function of the Reynolds number (dashed lines just connect the points).

is clear however, that filaments locally violate isotropy and the Kolmogorov scaling, and thus are likely to be a source of intermittency. We have shown that they occur at a smaller mean dimensionless frequency when the Reynolds number is increased. This is in agreement with the numerical simulations of reference [10] which show that the volume fraction occupied by the filaments decreases slowly when the Reynolds number is increased.

\section{Acknowledgements.}

We acknowledge B. Castaing and A. Pumir for useful discussions. This work has been supported by the EEC with contract CI1-CT91-0947.

\section{References}

[1] Willmarth W. W., Annu. Rev. Fluid Mech. 7 (1975) 13-38.

[2] George W. K., Beuther P. D. and Arndt R. E. A., J. Fluid Mech. 148 (1984) 155-191.

[3] Batchelor G. K., Proc. Cambridge Phil. Soc. 47 (1951) 359-374.

[4] Kraichnan R. H., J. Ac. Soc. Am. 28 (1956) 64-72, 378-390.

[5] Brachet M. E., Fluid Dyn. Res. 8 (1991) 1-8. 
[6] Fauve S., Laroche C. and Castaing B., J. Phys. II France 3 (1993) 271-278.

[7] Abry P. and Flandrin P., Progress in Wavelet Analysis and Applications, Y. Meyer and S. Roques Eds. (Toulouse, 1992) pp. 311-314.

[8] For a review, see Daubechies I., Ten lectures on wavelets SIAM (1992).

[9] Pumir A., A numerical study of pressure fluctuations in three-dimensional, incompressible, homogeneous, isotropic turbulence, submitted to Phys. Fluids (1993).

[10] Jimenez J., Wray A. A., Saffman P. and Rogallo R., J. Fluid Mech. 255 (1992) 65-90.

[11] Abry P., Goncalvès P. and Flandrin P., IEEE Int. Conf. on Acoustical, Speech and Signal Processing, ICASSP-93 (Mineapolis, 1992).

[12] Douady S., Couder Y. and Brachet M. E., Phys. Rev. Lett. 67 (1991) 983-986 .

[13] Métais O. and Lesieur M., J. Fluid Mech. 239 (1992) 157-194.

[14] Holzer M. and Siggia E., Phys. Fluids A 5 (1993) 2525-2532.

[15] Note that this cannot be an effect of the transducer being a low-pass filter. 